\title{
Metacognition, symptoms and general functioning in patients with schizophrenia
}

\author{
Maroua DAOUD, Jihene BEN THABET, Manel MAALEJ BOUALI, Sana OMRI, Rim FEKI, \\ Nacer ZOUARI, Lobna ZOUARI, , Nada CHARFI, Mohamed MAALEJ \\ Department of Psychiatry "C", Hedi Chaker University Hospital, Sfax,Tunisia
}

\section{Introduction and aim:}

- Poorer metacognitive abilities are recognized as strong predictors of social functioning deficits in individuals with schizophrenia.

- The aim of the current study was to examine metacognitive functioning in people with schizophrenia and to explore correlations between metacognition, symptoms and general functioning.

\section{Methods:}

- It was a cross-sectional study

- 74 outpatients, diagnosed with schizophrenia and followed in department of psychiatry "C" at Hedi Chaker university Hospital, in Sfax, participated in the study.

- Sociodemographic and clinical data were measured using selfreported questionnaires.

- The Positive and Negative Symptom Scale (PANSS) was developed to assess symptom severity in schizophrenia.

- The metacognition was assessed with the Metacognition Assessment Scale - Abbreviated version (MAS-A).

- The general functioning was measured with The Global Assessment of Functioning (GAF).

\section{Results:}

- The detail of basic demographics and scores of the MAS-A, and the GAF of the patients are shown in Table I.

- The results indicate that poor social functioning is associated with metacognitive difficulties (Table II).

- Greater metacognition was significantly correlated with fewer negative symptoms (Table II), but metacognition was not significantly correlated with positive psychotic symptoms, cognitive disorganisation, excitement or emotional distress.

\section{Discussion:}

- Our findings are consistent with previous work reporting that metacognition is impaired in patients with schizophrenia and tends to be a persistent feature in chronic patients (1)

- We sought to replicate previous research that has shown an association between metacognition and negative symptoms $(1,2)$. Interestingly, decentration emerged as the aspect of metacognition most strongly correlated with negative symptoms. One interpretation of this result is that negative symptoms are associated with increased self-focused attention with the accompanying distorted view that others are also focused on the patient. Difficulty perceiving events as unrelated to oneself may prompt the use of withdrawal and concealment strategies such as reducing affective expression, disengaging from others, and downregulating goal pursuit (i.e. affective blunting, emotional withdrawal, and avolition). We found a correlation between negative symptoms and the mastery scale, which indicates that worse negative symptoms were associated with greater difficulty using mental state information to solve interpersonal problems and challenges.

- In addition, studies consistently report a link between schizophrenia and social dysfunction, which has been attributed by some to the presence of metacognitive difficulties (3). The disturbances in social functioning are a result of metacognitive deficits or a failure to integrate information to form more complex ideas of themselves and others.

\section{Conclusion:}

- These findings underscore the importance of interventions designed to enhance the patients' metacognitive capacities, that is, the more proximal capacities linked to poorer social functioning.
TABLE I: Basic Demographics and MAS-A and GAF Scores

\begin{tabular}{|c|c|c|}
\hline & & Mean (SD) / N (\%) \\
\hline \multicolumn{2}{|l|}{ Age } & $34.1(11.8)$ \\
\hline \multicolumn{2}{|c|}{ Sex (men) } & $46(62.2 \%)$ \\
\hline \multirow[t]{5}{*}{ MAS-A } & Total score & $12.42(6.17)$ \\
\hline & Self-reflectivity “S” & $4.18(1.46)$ \\
\hline & $\begin{array}{l}\text { Understanding of others' } \\
\text { minds "O" }\end{array}$ & $3.20(1.06)$ \\
\hline & Decentration “D” & $2.5(1.8)$ \\
\hline & Mastery “M”' & 2.54 (1.85), \\
\hline \multicolumn{2}{|c|}{ GAF total score } & $49.39(10)$ \\
\hline
\end{tabular}

TABLE II: Correlation analysis between metacognition and a number of variables

\begin{tabular}{|c|c|c|c|c|}
\hline \multirow[t]{2}{*}{ Variable } & \multicolumn{4}{|c|}{ MAS-A (12.42 \pm 6.17$)$} \\
\hline & «S» & «U» & «D» & «M» \\
\hline General Functioning & \multicolumn{4}{|c|}{$0,822^{\star *}$} \\
\hline
\end{tabular}

(GAF)

\begin{tabular}{lllll} 
Negative symptoms & $-0,37^{*}$ & $-0,52^{*}$ & $-0,24^{*}$ & $-\mathbf{0 ,} 7^{* *}$ \\
(PANSS) & & & & \\
\hline${ }^{*} \mathrm{p}<0,05 ; \quad{ }^{*} \mathrm{p}<0,01$ &
\end{tabular}

